\title{
COLD AGGLUTININS. I. OCCURRENCE OF COLD ISOHEMAGGLUTININS IN VARIOUS CONDITIONS
}

\author{
By MAXWELl FINLAND, OSLER L. PETERSON, HOWARD E. ALLEN, \\ BERNARDO A. SAMPER AND MILDRED W. BARNES, wITH THE \\ TECH NICAL ASSISTANCE OF MURIEL B. STONE \\ (From the Thorndike Memorial Laboratory, Second and Fourth Medical Services (Harvard), \\ Boston City Hospital, and the Department of Medicine, Harvard \\ Medical School, Boston)
}

(Received for publication September 22, 1944)

The development of significant titers of cold agglutinins (autohemagglutinins) in most cases of primary atypical pneumonia of unknown eti$\operatorname{ology}^{1}(1,2)$ was first noted in this laboratory (3) and later, independently elsewhere (4 to 6). This finding has since been confirmed in other clinics $(7,8)$. A few cases of pneumonia are included among the previously reported cases of marked autohemagglutination collected from the literature in 1937 (9) and a detailed study of some of the important properties of the cold agglutinin was carried out in a case of "diffuse bronchopneumonia" reported in 1918 (10). Cold agglutinins have also been described in many other conditions, notably in association with various blood dyscrasias, liver diseases, and in allergic and peripheral vascular disorders. Their occurrence even in these conditions is still sufficiently rare that only individual cases or very small groups of cases have been reported by any single observer. The only infectious disease in which autoagglutinins have been found with any great regularity is trypanosomiasis (11)

The literature concerning the occurrence of cold agglutinins, their nature and properties, and the methods for detecting them have been reviewed recently (12). Very few reports are available of extensive surveys of the occurrence of cold agglutinins in various infectious diseases with the use of standardized quantitative methods. In a study of sera from 421 cases of various common infectious diseases (6), significant titers were found only in cases of primary atypical pneumonia and of mumps complicated with orchitis. Many of the sera included in that study had been stored for several months. Other authors $(7,8)$ obtained negative results, in 135 and in 138 control

\footnotetext{
1 Hereafter referred to as "atypical pneumonia."
}

cases respectively, including normals and persons with various infections other than primary atypical pneumonia.

The present paper deals with the results of tests for cold agglutinins in 1069 cases of a variety of common diseases particularly those involving infections of the respiratory tract. Details concerning the occurrence of cold agglutinins in the 200 characteristic cases of primary atypical pneumonia included among these cases and the results of studies of some of the properties of the cold agglutinins are left for separate consideration in the papers which follow.

\section{MATERIALS AND METHODS}

Patients. Except for some of the patients with atypical pneumonia from other hospitals, almost all of the cases studied were regular admissions to the wards of the Boston City Hospital between September 1942 and March 1944. Control sera included a number of serial specimens provided by Drs. T. Duckett Jones and Benedict F. Massell, from carriers and non-carriers of hemolytic streptococci, as well as from 6 cases of rheumatic fever. Many of these had hemolytic streptococcal infections shortly before or during the period when the bloods were being collected. Many cases were chosen specifically for this study as representatives of disease entities, others were selected because they had febrile illnesses of more than a few days duration while still others were included because they were receiving sulfonamide therapy for a week or longer. Almost all of the patients with bacterial pneumonias and about two-thirds of the remaining cases, other than those without disease, received sulfonamide therapy. The diagnosis recorded in each case was arrived at by the authors after reviewing all of the available data.

Both serum and plasma were used in the tests. Venous blood was obtained in most cases under sterile precautions and allowed to clot at room temperature or at $37^{\circ} \mathrm{C}$. The clear serum was then separated and removed after centrifugation. Some of the tests were carried out immediately but usually the sera were stored in sterile rubber stoppered test tubes in a refrigerator at 5 to $10^{\circ} \mathrm{C}$. and the tests were carried out in most instances within a 
TABLE I

Cold isoagglutinins in various conditions

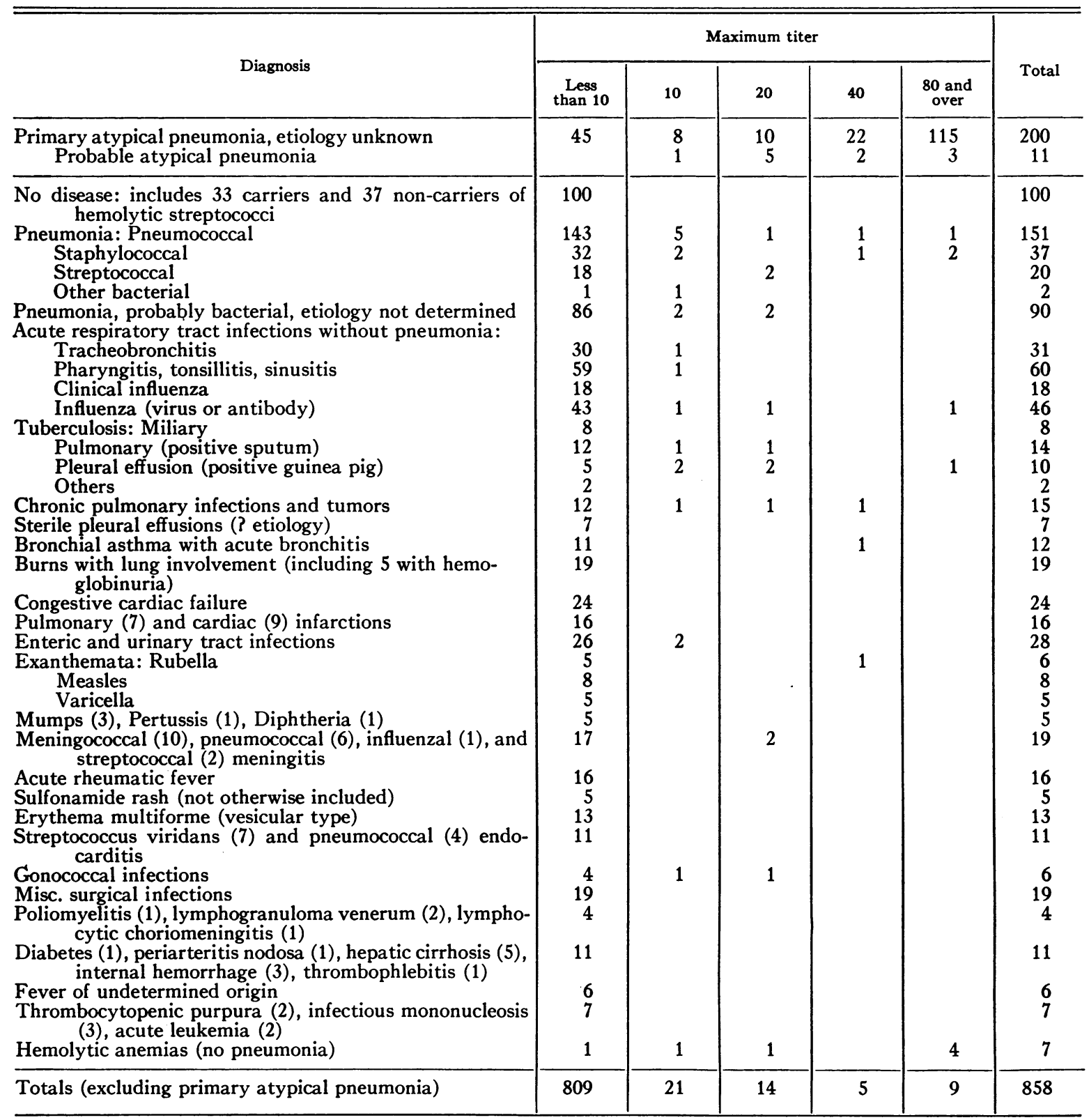

few days. A number of specimens were obtained as oxalated blood. These were warmed to $37^{\circ} \mathrm{C}$. and the plasma, separated by centrifugation, was used for the tests on the day it was obtained or on the following day. Serial specimens were taken at suitable intervals in most of the cases but single samples were obtained in some of them, usually during the second to the fourth week after onset of the febrile illness.

The definition of cold agglutinins for purposes of this study is the one usually accepted for human bloods. The agglutination is independent of the blood groups, is optimum at 0 to $5^{\circ} \mathrm{C}$., diminishes with rise in temperature, and is no longer demonstrable at $37^{\circ} \mathrm{C}$. For convenience, the tests were done for cold isoagglutinins rather than for autoagglutinins which are usually, though not always, entirely comparable. The erythrocytes in these tests were obtained from group "O" donors. A few members of the laboratory staff contributed all of the blood used and most of the cells were obtained from 3 of these donors. Oxalated blood was washed 3 times in a large volume of 
saline and a 2 per cent suspension of cells in physiological saline was then made and used in the tests. For the present study, almost all of the tests were carried out with cells that were 2 to 4 days old.

The tests for cold agglutinins were carried out as follows: Serial 2-fold dilutions of serum or plasma in saline were made in $0.5 \mathrm{ml}$. amounts in $100 \times 11 \mathrm{~mm}$. test tubes. In most of the early tests, the first dilution was $1: 2$ but in the later ones it was 1:5. Equal volumes of 2 per cent cells were added, the tubes were shaken thoroughly, and stored in a refrigerator overnight. The racks of tubes were usually placed in an ice water bath before the readings were made. In reading the tests, the tubes were rapidly inverted 3 times to loosen all of the cells from the bottom of the tube and to allow thorough mixing. The agglutination was graded from $4+$ to $1+$, the former representing a single hard clump of cells not broken up on mixing while the latter indicated a finely floccular agglutination, easily visible without magnification. Finer agglutination, visible only with the aid of a magnifying lens $( \pm)$, was noted but not considered in the values recorded. The titers are recorded as the reciprocal of the highest final dilution of serum giving $1+$ agglutination.

After the readings were made in the cold, the racks of tubes were placed in a water bath or incubator at $37^{\circ} \mathrm{C}$. for 2 hours. All the positive agglutinations recorded in these studies were completely reversed by this procedure.

\section{RESULTS}

A summary of the maximum titers obtained in the 1069 cases of various diseases is given in Table I. It is seen at a glance that two groups of cases stand out from among all the others. They are the cases of atypical pneumonia and those of hemolytic anemia. Of the 200 characteristic cases of atypical pneumonia, 137 or 68.5 per cent had a maximum titer of 40 or higher. The cases listed as "probable atypical pneumonia" each had a similar characteristic clinical course but either the lung lesion had already cleared when the first specimen of blood was obtained, or the history and physical signs were characteristic, but the $x$-ray was negative, or complicating factors such as congestive heart failure or superimposed bacterial infections made the interpretation of the pulmonary findings uncertain. All of these cases had cold agglutinins, but a titer of 40 or higher was obtained in only 5 of them.

Among the cases other than those of atypical pneumonia, cold agglutinins were found in the greatest frequency and highest titers in the hemolytic anemias. Significant titers were obtained in 4 of 7 such cases and low titers (10 or 20) were obtained in 2 of the others. The bloods in all of these cases were studied by Dr. T. Hale Ham in considerable detail. A few of the significant facts in the 4 cases with the high titers may be noted.

The first of these was in a colored man of 35 who was first studied by Dr. Ham in 1939. At that time he had a Lederer's type of acute hemolytic anemia with cold agglutinins in a titer of $160(1+)$ and strong $(2$ or $3+$ ) agglutination in the lower dilutions. The patient had latent syphilis at the time and more than 2 years later he was treated in the hospital for luetic meningitis, but his anemia had cleared by then and he no longer had cold agglutinins.

The second patient was a colored man, 45 years old, who was first admitted on February 12, 1943, and was found at that time to have hypertensive heart disease, syphilitic laryngitis, and an acute upper respiratory tract infection. At this time, the patient may have had atypical pneumonia which was not recognized because of the other findings. No abnormal blood cytological findings were made out during this admission and tests for cold agglutinins were not done. On March 11, 2 weeks after discharge, he was readmitted because of hemoglobinuria and severe anemia. The cold agglutinin titer was 1280 on March 11 and dropped to 320 during the next 6 weeks. The agglutination was marked $(3+$ and $4+)$ in all but the highest dilutions. The anemia improved and the patient was discharged but he again returned $21 / 2$ months later, at which time he died of congestive heart failure without a recurrence of the anemia. The cold agglutinin titer at this time, however, was $2560(2+)$ and again the agglutination was very marked $(4+$ and $3+)$ in all but the highest dilution.

The third case was that of a woman, 45 years old, who had long suffered from cold urticaria and hemoglobinuria. In this case, too, the cold agglutinin titer was 2560 with marked agglutination occurring in all but the last dilution of serum.

The fourth case was in a colored man of 34 who developed a marked acute hemolytic anemia on the sixth day of treatment with sulfanilamide for acute glomerular nephritis and streptococcal pharyngitis. Cold agglutinin titers of 40 and 80 were obtained in 2 samples of serum on the day after the anemia and hemolysis occurred and after the sulfanilamide was discontinued. Three weeks later, the titer was still 80 . Strong agglutination ( $3+$ and $2+$ ) occurred in the $1: 10$ and $1: 20$ dilutions in each instance.

In all of these 4 cases, there was increased osmotic fragility of the red blood cells (only a slight increase was demonstrated on the first day in the last case mentioned), the Donath-Lansteiner and acid hemolysis tests (13) were negative, and the cold agglutination was completely abolished at $37^{\circ} \mathrm{C}$. 
Among the remaining 851 cases, only 10 , or 1.2 per cent, had cold agglutinins in titers of 40 or higher. A few pertinent remarks concerning the illness and the course and character of the cold agglutination observed in these cases are also of interest. There were 5 such cases among those listed as bacterial pneumonias.

One of these cases was in a man 42 years old, who had an abrupt onset of typical lobar pneumonia on March 2, 1943, without antecedent upper respiratory tract infection. He was admitted on the following day and received full doses of sulfapyrazine for 6 days. His temperature fell to normal on the second day, only to return almost to the original level of $104^{\circ}$ on the following day and the fever persisted until the tenth day. During this time, there were physical and $x$-ray signs first of frank consolidation of the left upper lobe and then of atelectasis of that lobe followed by extension of the consolidation to the lower lobe. The leukocyte counts varied from 27,000 to 11,000 during the febrile course. Type 7 pneumococci predominated in cultures of the sputum obtained at entry and repeated blood cultures were negative. A cold agglutinin titer of $40(1+)$ was obtained on the seventh day and a slightly stronger agglutination $(2+)$ was noted only in the $1: 10$ dilution of the same serum. The second case was one of a type 7 pneumococcal pneumonia with negative blood cultures, leukocytosis, and a good response to penicillin. A titer of 160 was obtained both in acute-phase and convalescent sera. The patient had a severe cough for 3 weeks prior to the abrupt onset of the lobar pneumonia.

In the other 3 cases, staphylococcus aureus was the only or predominant organism. The first of these was in a woman 22 years old whose illness began on September 22, 1943, and was characterized by chilliness, substernal and right lower chest pain, cough, and purulent sputum. There were physical and $\mathbf{x}$-ray signs of dense consolidation of the left lower lobe and possibly of fluid, but the latter was not demonstrated by thoracentesis. Sputum and blood cultures obtained on the fifth and ninth days were positive for hemolytic staphylococcus aureus. The leukocyte count stayed between 8500 and 9400 throughout the febrile course. There was gradual improvement in the fever and symptoms on sulfamerazine therapy. Drug therapy was discontinued after 10 days because of the occurrence of a rash and fever. The cold agglutinin titer was $40(2+)$ on the fourteenth day and $80(2+)$ on the twenty-first day. The early clinical course in this case strongly suggested that of a primary atypical pneumonia with superimposed staphylococcal infection.

In the second case of staphylococcal pneumonia there were a number of complicating factors. The patient was a woman 56 years old who was admitted on February 10, 1943 , with a variety of symptoms which, together with the physical and laboratory findings, were interpreted as those of cirrhosis of the liver and chronic lymphogranuloma venereum. There were scattered medium moist râles, and $\mathbf{x}$-rays showed fine miliary nodular areas of density throughout both lung fields. The patient had a persistent low grade fever and leukopenia. She also had an active osteomyelitis of a finger and staphylococcus aureus septicemia. The pulmonary lesions persisted throughout her stay. She died after 7 weeks in the hospital and autopsy was not obtained. On 2 occasions in the middle of her hospital course, her blood showed cold agglutinins in a titer of $80(1+)$ with somewhat stronger agglutination $(2+)$ in all the lower dilutions. In this case, also, the possibility that the pulmonary lesion began as a primary atypical pneumonia cannot be entirely excluded.

The last case among the pneumonias was in a man of 64. He was admitted in October 1943 on the third day of an illness which began with chills, diarrhea, headache, pleuritic pain in the left lower chest, dyspnea, and cough productive of grossly bloody sputum. He had had a slight coryza 3 days prior to the onset of these symptoms. There were physical and $\mathbf{x}$-ray signs of consolidation of the left lower lobe. The leukocyte counts ranged between 3000 and 7100. Staphylococcus aureus predominated in cultures of the sputum, and the blood cultures were all negative. On sulfamerazine therapy, the patient's temperature dropped in 2 days from 102.8 to about $100^{\circ} \mathrm{F}$. and a low grade fever persisted for another week. In this case, a cold agglutinin titer of $40(1+)$ was noted on the sixteenth day but there was no stronger agglutination observed in any of the lower dilutions of the same serum.

One patient with tuberculous pleurisy with effusion had cold agglutinins. He was a man of 40 who had had a slight cough for a year until one week prior to his entry on February 22, 1944, when he began to have chilly sensations, sweats, and substernal pain. Characteristic fluid was obtained which yielded tubercle bacilli on guinea pig inoculation. Fever reaching $102^{\circ} \mathrm{F}$. daily persisted throughout his hospital stay, during which he received 2 courses of sulfapyrazine therapy. The cold agglutinin titer before sulfonamide therapy was started was $\mathbf{8 0}$ $(1+)$ with somewhat stronger agglutination $(2+)$ in the lower dilutions. After the first course of sulfapyrazine, the titer was $40(1+)$. In this case, too, the early symptoms preceding the onset of the effusion suggest the possibility that the patient had atypical pneumonia which activated a pre-existing tuberculosis. Similar reactivation of tuberculosis has been observed in this clinic following definite influenza virus infections. It is of interest that all 4 of the other cases of tuberculous pleurisy with effusion which were studied showed some cold agglutinins in titers of 10 and 20 . In each of these cases, however, the agglutination was weak $(1+)$ even in lower dilutions.

One patient with a proved carcinoma of the lung had a cold agglutinin titer of 40 . In this case, however, the agglutination was weak $(1+)$ in all the dilutions that were tested. The patient's symptoms consisted of cough, bloody sputum, anorexia, and loss of weight. Signs of fluid predominated in the affected side of the chest and characteristic bloody fluid was removed and shown to contain tumor cells.

The case of rubella was a typical one with a slightly 
prolonged febrile course. Cold agglutinins in a titer of $40(1+)$ were demonstrated in serum obtained several days after the fever and rash had subsided. The agglutination was somewhat stronger $(2+)$ in the lower dilution of the same serum. There was no clinical evidence of pneumonia in this case except for a slight cough.

The patient with bronchial asthma was a man of 70 who was admitted February 24, 1943, two days after the onset of an acute tracheobronchitis. There were scattered crepitant as well as musical râles throughout the chest and physical and $x$-ray signs of bronchiectasis and of patchy bronchopneumonia in both lower lobes. He had a febrile course of 11 . days in spite of sulfadiazine therapy. Blood taken on the eighth day had a cold agglutinin titer of $40(1+)$ and the agglutination was weak $(1+)$ in the lower dilutions as well.

The cases of influenza are of special interest. Of the 64 cases studied, all except 2 of those from which virus was isolated occurred during the course of the epidemic in December 1943 and early in 1944 (14). Serial samples of serum were tested in most of these cases. In 2 cases, there was weak cold agglutination $(1+)$ in titers of 10 or 20 and the same titers were observed in all the sera from each case. In only one case did cold agglutinins develop to a significant titer.

This case was in an intern who had the characteristic symptoms of clinical influenza which began February 26 , 1944 , several weeks after the peak of the influenza epidemic in Boston. He had been in Minnesota during the previous November and December when the epidemic of influenza was at its height there but he had no symptoms at that time. His clinical course was characterized by daily swings in temperature from normal to $101^{\circ} \mathrm{F}$. for 4 days, with cough and headache throughout this time. There were a few scattered fine râles heard only in his right lower lobe for several days but repeated $x$-rays revealed no abnormal findings in the lungs. The leukocyte counts remained normal throughout his course. A few pneumococci, Type 28, were obtained in cultures of throat washings obtained for virus studies on the day after the onset. These washings were injected intranasally in mice and into the allantoic sac of 11-day chick embryos and readily yielded a virus which had many of the characteristics of an influenza virus but it differed serologically from typical strains of both $A$ and $B$ influenza viruses. This strain is now being studied further. No rise in antibodies to either the PR8 (type A) or the Lee (type B) strains of influenza could be demonstrated by either the complement fixation or the agglutination-inhibition (Hirst) tests and no rise was demonstrated by the latter test against his own virus. ${ }^{2}$ The sera obtained on the .second and ninth days showed no cold agglutinins but those of the sixteenth and thirty-second days had titers of

\footnotetext{
2 This patient is listed as Case 68 in reference 14.
}

$20(2+)$ and $80(2+)$, respectively. Such titers have been obtained at corresponding stages of the disease in many characteristic but mild cases of primary atypical pneumonia.

From the data presented, it is apparent that among the cases other than those of hemolytic anemia, some at least may have had atypical pneumonia. In them, either positive $x$-ray signs were not made out or the symptomatology and physical signs were obscured by those of other coexisting diseases. Even in one of the cases of hemolytic anemia,- the one which was complicated by syphilis and hypertensive heart disease,-the appearance of or increase in cold agglutinins may very well have resulted from the acute respiratory infection which occasioned the first entry. This infection, in turn, had many characteristics of atypical pneumonia, but, unfortunately, studies with this in mind were not made until the patient was readmitted later with hemoglobinuria and anemia. In fact, $x$-rays taken for other purposes during the first admission showed suggestive lesions in the lungs which had been overlooked. The signs of atypical pneumonia, if that is what he had, were completely overshadowed by those of syphilitic laryngitis and congestive failure.

\section{DISCUSSION}

The data presented indicate quite clearly that significant titers of cold agglutinins, demonstrable by the method used, are found infrequently in normal individuals and in patients with respiratory or other infectious diseases except in cases of primary atypical pneumonia and in certain types of hemolytic anemia. Indeed, in some of the few other cases in which similar titers of cold agglutinins were demonstrated, the possibility of recent or coexisting atypical pneumonia could not be entirely ruled out. In fact, a detailed review of the other cases in which cold agglutinin titers of 10 or 20 were found in this study showed a similar likelihood of the coexistence of atypical pneumonia in many of them. From the literature it would appear that only in cases of trypanosomiasis have cold agglutinins been encountered with the same regularity. ${ }^{3}$ Furthermore, a careful study of

\footnotetext{
3 A high incidence of cold agglutinins has been reported recently in cases of tropical eosinophilia (16) and among Melanesian natives in yaws epidemic areas (17).
} 
the previously reported cases of marked autohemagglutination suggests that many of them may have had pulmonary infections similar to those which are currently being classified as atypical pneumonia. Favour (15), however, found cold agglutinins more frequently in other conditions.

At this time, it is still reasonable to say that no single specific etiological agent has as yet been identified in cases of primary atypical pneumonia and consequently no reliable immunological test based on such a specific etiological agent is available for the definite identification and classification of such cases. The development of cold agglutinins may, therefore, serve as a helpful diagnostic aid in these cases until such agent or agents are discovered. At least the development of cold agglutinins in patients presenting characteristic symptoms and physical and $x$-ray signs may serve to segregate one group of cases among those now classified as primary atypical pneumonia of unknown etiology. It also seems reasonable to assume that the development of cold agglutinins in these cases is related in some way to the unknown causative agent. This does not entirely preclude the possibility that other cases having the same etiological agent may run a similar clinical course without developing cold agglutinins and, conversely, that certain other types of etiological agents may produce cold agglutinins. The relation to the disease or to the development of cold agglutinins of the virus isolated from the patient who was considered, on clinical grounds, as having influenza is still obscure.

In this study, the tests for cold agglutinins were carried out in each case within a few days of the time when the blood was drawn. This is an important factor, particularly in sera of low titer as will be shown in a later paper in this series. Deterioration on storage of the cold agglutinins in sera of atypical pneumonias was postulated by Horstmann and Tatlock (7) because of the significantly lower titers found by them in sera that had been stored for a long time before they were tested.

\section{SUMMARY AND CONCLUSIONS}

Tests for cold agglutinins, using erythrocytes from normal group 0 donors, were carried out in 1069 cases, including normal persons and patients with a variety of conditions, mostly respiratory tract infections of various sorts.

Cold agglutinins in titers of 40 or higher were found in 137 or 68.5 per cent of 200 characteristic cases of primary atypical pneumonia of unknown etiology, in 5 of 11 cases in which this was the probable diagnosis, and in 4 of 7 cases of various kinds of hemolytic anemia.

In only 10 , or 1.2 per cent of the remaining 851 cases were cold agglutinins demonstrated in similar titer. In some of these 10 cases and of the few others in which cold agglutinins were demonstrated in lower titers, atypical pneumonia may have played a part.

It is suggested that the development of cold agglutinins is related to the causative agent of primary atypical pneumonia and may serve as a diagnostic aid in this disease.

The authors are deeply indebted to the many physicians, both at the Boston City Hospital and elsewhere, who permitted us to see and follow their cases, obtained the blood samples, and made available the clinical and laboratory data. Dr. T. Hale Ham provided some of the sera and studied the blood in the cases of hemolytic anemia and Miss Geneva A. Daland carried out some of the tests in these cases.

\section{BIBLIOGRAPHY}

1. Official Statement, Primary atypical pneumonia, etiology unknown. War Med., 1942, 2, 330.

2. Dingle, J. H., and Finland, M., Virus pneumonias. II. Primary atypical pneumonias of unknown etiology. New England J. Med., 1942, 227, 378.

3. Peterson, O. L., Ham, T. H., and Finland, M., Cold agglutinins (autohemagglutinins) in primary atypical pneumonias. Science, 1943, 97, 167.

4. Turner, J. C., Development of cold agglutinins in atypical pneumonia. Nature, 1943, 151, 419.

5. Turner, J. C., and Jackson, E. B., Serological specificity of an auto-antibody in atypical pneumonia. Brit. J. Exper. Path., 1943, 24, 121.

6. Turner, J. C., Nisnewitz, S., Jackson, E. B., and Berney, R., Relation of cold agglutinins to atypical pneumonia. Lancet, 1943, 1, 765.

7. Horstmann, D. M., and Tatlock, H., Cold agglutinins : a diagnostic aid in certain types of primary atypical pneumonia. J. A. M. A., 1943, $122,369$.

8. Meiklejohn, G., The cold agglutination test in the diagnosis of primary atypical pneumonia. Proc. Soc. Exper. Biol. and Med., 1943, 54, 181.

9. McCombs, R. P., and McElroy, J. S., Reversible autohemagglutination with peripheral vascular symptoms. Arch. Int. Med., 1937, 59, 107.

10. Clough, M. C., and Richter, I. C., A study of an auto- 
agglutinin occurring in a human serum. Bull. Johns Hopkins Hosp., 1918, 29, 86.

11. Yorke, W., Autoagglutination of the red blood cells in trypanosomiasis. Ann. Trop. Med., and Parasitology, 1910, 4, 529.

12. Stats, D., and Wasserman, L. R., Cold hemagglutination-an interpretive review. Medicine, 1943, 22, 363.

13. Ham, T. H., Studies on the destruction of red blood cells. Chronic hemolytic anemia with paroxysmal nocturnal hemoglobinuria. An investigation of the mechanism of hemolysis. Arch. Int. Med., 1939, 64, 1271.
14. Finland, M., Barnes, M. W., and Samper, B. A., Influenza virus isolations and serological studies made in Boston during the winter of 1943-1944. J. Clin. Invest., 1945, 24, 192.

15. Favour, C. B., Auto-hemagglutinins-cold agglutinins. J. Clin. Invest., 1944, 23, 891.

16. Visivanathan, R., and Natarajori, B., Cold agglutinins in tropical eosinophilia. Lancet, 1945, 1, 148.

17. Fetterman, G. H., Moran, T. J., and Hess, W. R., The cold agglutination test: I. Studies on Naval Hospital patients; II. Studies on natives in yawsepidemic areas. U. S. Nav. M. Bull., 1944, 43, 1128. 